\title{
Linkage studies in a pedigree with Van der syndrome
}

\author{
THOMAS F WIENKER*, GÜNTHER HUDEK*, SIEGBERT BISSBORT*, \\ ANTONIA MAYEROVÁ*, GOTTFRIED MAUFF†, AND KLAUS BENDER* \\ From * Institut für Humangenetik und Anthropologie der Universität Freiburg, D-7800 Freiburg; and \\ †Hygiene-Institut der Universität Köln, D-5000 Köln 41, West Germany.
}

SUMMARY A kindred segregating for Van der Woude syndrome (VWS) through five generations is described. Biochemical and serological phenotypes at 36 polymorphic marker loci have been determined, of which 27 were informative for linkage analysis to the VWS gene (LIPED 8 computer programme). Lod scores are reported and show exclusion of close linkage for most of the marker loci. Only $V W S: D u f f y(F y)$ resulted in uniformly positive lod scores $(\hat{\theta}=0 \cdot 0 \overrightarrow{,}$ $\mathrm{z}(\hat{\theta})=1 \cdot 31)$.

Van der Woude syndrome (VWS) is a rare genetic malformation syndrome characterised by one or more of three primary manifestations: (1) bilateral lower lip pits, (2) cleft lip with or without cleft palate, or (3) isolated cleft palate. It is inherited as an autosomal dominant trait with 70 to $80 \%$ penetrance suggested by early studies, ${ }^{1-2}$ although Janku $e t a l^{3}$ have reported a higher penetrance of about $97 \%$. The lower penetrance reported in earlier papers on this syndrome is most probably due to incomplete ascertainment, that is, nonrecognition of patients with 'microforms', and true penetrance is probably close to $100 \%$. The chromosomal location of the VWS gene is not known. Three pedigrees have been analysed for linkage relations so far, ${ }^{4-6}$ but only the data of Eastman et $a l^{5}$ were reported in detail. No evidence of close linkage was found between the VWS gene and any segregating marker locus in these studies. We had the opportunity to study another large pedigree with Van der Woude syndrome and report here the results of linkage analysis using 36 marker loci.

\section{Subjects and methods}

This family came to our attention when an affected member (IV.7, figure) requested genetic counselling. All living members reside in rather isolated conditions in the southern part of the Black Forest. They are in touch with one another and know their ancestry well. All those interviewed knew the

Received for publication 3 July 1986.

Revised version accepted for publication 22 August 1986. 'family mark' and were remarkably accurate in their description of pits and clefts, although they could rarely give information about missing teeth (hypo dontia). The pedigree, spanning five generations comprises a total of 116 members and has beern presented in full by Hudek. ${ }^{7}$ Only 19 of the livingD members and four spouses were cooperative and they were examined for the presence of VWS
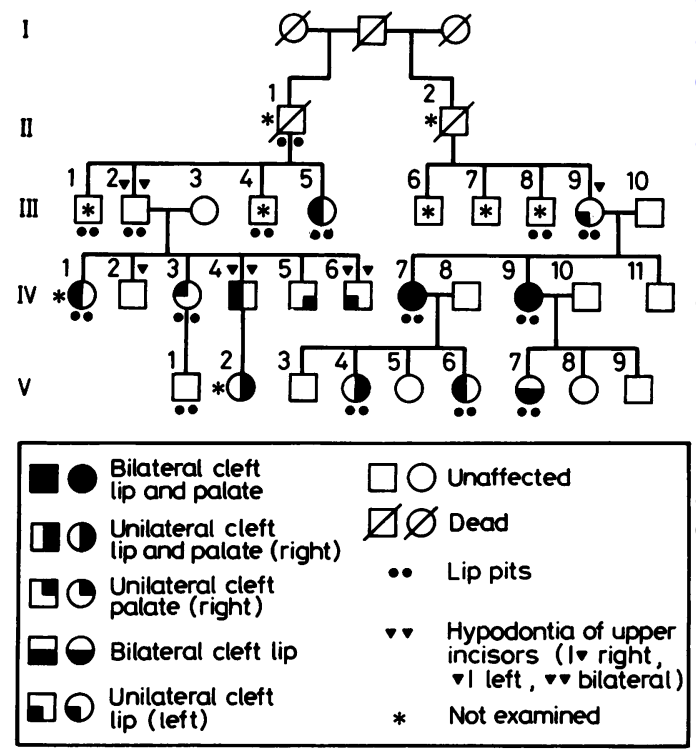

FIGURE Pedigree of family. 
TABLE Linkage relations of the VWS gene.

\begin{tabular}{|c|c|c|c|c|c|c|c|c|c|c|c|c|c|}
\hline \multirow{2}{*}{$\begin{array}{l}\text { Locus } \\
\text { (chromosome } \\
\text { assignment) }\end{array}$} & \multirow[t]{2}{*}{ Sex } & \multicolumn{5}{|c|}{ Lod scores at recombination fraction $(\theta)$} & \multirow{2}{*}{$\begin{array}{l}\text { Locus } \\
\text { (chromosome } \\
\text { assignment) }\end{array}$} & \multirow[t]{2}{*}{ Sex } & \multicolumn{5}{|c|}{ Lod scores at recombination fraction $(\theta)$} \\
\hline & & 0.05 & $0 \cdot 1$ & $0 \cdot 2$ & $0 \cdot 3$ & 0.4 & & & 0.05 & $0 \cdot 1$ & $0 \cdot 2$ & $0 \cdot 3$ & $0 \cdot 4$ \\
\hline $\begin{array}{l}R h \\
(1 \mathrm{p} 36 \cdot 2 \rightarrow \mathrm{p} 34)\end{array}$ & $\begin{array}{l}\text { M } \\
\text { F } \\
\text { Sum* }\end{array}$ & $\begin{array}{l}-2 \cdot 16 \\
-1 \cdot 74 \\
-2 \cdot 70\end{array}$ & $\begin{array}{l}-1 \cdot 33 \\
-1 \cdot 16 \\
-1 \cdot 65\end{array}$ & $\begin{array}{l}-0.58 \\
-0.61 \\
-0.67\end{array}$ & $\begin{array}{l}-0 \cdot 23 \\
-0 \cdot 31 \\
-0 \cdot 28\end{array}$ & $\begin{array}{l}-0.05 \\
-0.12 \\
-0.11\end{array}$ & $\begin{array}{l}P L G \\
(6 \mathrm{q} 25 \rightarrow \mathrm{qter}) \\
A B O\end{array}$ & $\mathbf{F}$ & -1.65 & $-1 \cdot 07$ & -0.48 & $-0 \cdot 19$ & $-0 \cdot 04$ \\
\hline $\begin{array}{l}P G M 1 \\
(1 \mathrm{p} 22 \cdot 1)\end{array}$ & $\begin{array}{l}\mathbf{M} \\
\mathbf{F} \\
\text { Sum* }\end{array}$ & $\begin{array}{l}-1 \cdot 39 \\
-0 \cdot 98 \\
-3 \cdot 26\end{array}$ & $\begin{array}{l}-0 \cdot 86 \\
-0 \cdot 66 \\
-2 \cdot 08\end{array}$ & $\begin{array}{l}-0.39 \\
-0.37 \\
-1.76\end{array}$ & $\begin{array}{l}-0 \cdot 16 \\
-0 \cdot 18 \\
-0.87\end{array}$ & $\begin{array}{l}-0.04 \\
-0.07 \\
-0.15\end{array}$ & $(9 q 34)$ & $\begin{array}{l}\text { F } \\
\text { Sum* }\end{array}$ & $\begin{array}{l}-0.29 \\
-0.77\end{array}$ & $\begin{array}{l}-0.25 \\
-0.29\end{array}$ & $\begin{array}{l}-0 \cdot 17 \\
+0 \cdot 07\end{array}$ & $\begin{array}{l}-0.11 \\
+0.09\end{array}$ & $\begin{array}{l}-0 \cdot 05 \\
+0 \cdot 02\end{array}$ \\
\hline $\begin{array}{l}F y \\
(1 \mathrm{p} 21 \rightarrow \mathrm{q} 23)\end{array}$ & $\begin{array}{l}\mathbf{M} \\
\text { F } \\
\text { Sum* }\end{array}$ & $\begin{array}{l}+0 \cdot 13 \\
+1.08 \\
+1.08\end{array}$ & $\begin{array}{l}+0 \cdot 10 \\
+0 \cdot 98 \\
+1 \cdot 12\end{array}$ & $\begin{array}{l}+0.06 \\
+0.78 \\
+0.98\end{array}$ & $\begin{array}{l}+0.03 \\
+0.56 \\
+0.72\end{array}$ & $\begin{array}{l}+0.01 \\
+0.30 \\
+0.38\end{array}$ & $\begin{array}{l}E S D \\
(13 \mathrm{q} 14 \cdot 1) \\
P I \\
(14 \mathrm{q} 32 \cdot 1)\end{array}$ & $\mathbf{M}$ & $-1 \cdot 23$ & $-0 \cdot 72$ & $-0 \cdot 30$ & $-0 \cdot 11$ & $-0 \cdot 03$ \\
\hline $\begin{array}{l}A C P 1 \\
(2 \mathrm{p} 25 \text { or } 2 \mathrm{p} 23)\end{array}$ & $\begin{array}{l}\text { M } \\
\text { F } \\
\text { Sum* }\end{array}$ & $\begin{array}{l}-0 \cdot 16 \\
-1 \cdot 19 \\
-0 \cdot 94\end{array}$ & $\begin{array}{l}-0 \cdot 12 \\
-0 \cdot 88 \\
-0 \cdot 70\end{array}$ & $\begin{array}{l}-0.06 \\
-0.49 \\
-0.35\end{array}$ & $\begin{array}{l}-0 \cdot 03 \\
-0 \cdot 26 \\
-0 \cdot 18\end{array}$ & $\begin{array}{l}-0.01 \\
-0 \cdot 10 \\
-0.07\end{array}$ & $\underset{(14 q 32 \cdot 3)}{G m}$ & $\begin{array}{l}\text { M } \\
\text { F } \\
\text { Sum }\end{array}$ & $\begin{array}{l}-1.40 \\
-2.13 \\
-3.53\end{array}$ & $\begin{array}{l}-0.85 \\
-1 \cdot 50 \\
-2 \cdot 35\end{array}$ & $\begin{array}{l}-0.36 \\
-0.80 \\
-1 \cdot 16\end{array}$ & $\begin{array}{l}-0.14 \\
-0.40 \\
-0.54\end{array}$ & $\begin{array}{l}-0 \cdot 03 \\
-0 \cdot 15 \\
-0 \cdot 18\end{array}$ \\
\hline $\begin{array}{l}J K \\
(2)\end{array}$ & $\begin{array}{l}\mathbf{M} \\
\mathbf{F} \\
\text { Sum* }\end{array}$ & $\begin{array}{l}+0.07 \\
-0.46 \\
-0.07\end{array}$ & $\begin{array}{l}+0 \cdot 05 \\
-0 \cdot 32 \\
-0 \cdot 05\end{array}$ & $\begin{array}{l}+0 \cdot 03 \\
-0 \cdot 15 \\
+0 \cdot 01\end{array}$ & $\begin{array}{l}+0.01 \\
-0.06 \\
+0.02\end{array}$ & $\begin{array}{r}0.00 \\
-0 \cdot 01 \\
+0 \cdot 01\end{array}$ & $\begin{array}{l}H P \\
(16 \mathrm{q} 22 \cdot 1)\end{array}$ & $\begin{array}{l}\text { M } \\
\text { F } \\
\text { Sum* }\end{array}$ & $\begin{array}{l}-0.65 \\
-1 \cdot 13 \\
-1 \cdot 26\end{array}$ & $\begin{array}{l}-0.39 \\
-0.71 \\
-0.69\end{array}$ & $\begin{array}{l}-0 \cdot 16 \\
-0 \cdot 25 \\
-0 \cdot 12\end{array}$ & $\begin{array}{l}-0.06 \\
-0.04 \\
+0.08\end{array}$ & $\begin{array}{l}-0 \cdot 01 \\
+0 \cdot 04 \\
+0 \cdot 11\end{array}$ \\
\hline $\begin{array}{l}T F \\
(3 \mathrm{q} 21 \rightarrow \mathrm{q} 26 \cdot 1)\end{array}$ & $\begin{array}{l}\mathbf{M} \\
\mathbf{F} \\
\text { Sum }\end{array}$ & $\begin{array}{l}-0 \cdot 05 \\
-1 \cdot 18 \\
-1 \cdot 23\end{array}$ & $\begin{array}{l}-0 \cdot 04 \\
-0 \cdot 80 \\
-0 \cdot 84\end{array}$ & $\begin{array}{l}-0 \cdot 02 \\
-0.38 \\
-0.40\end{array}$ & $\begin{array}{l}-0 \cdot 01 \\
-0 \cdot 16 \\
-0 \cdot 17\end{array}$ & $\begin{array}{r}0.00 \\
-0.04 \\
-0.04\end{array}$ & $\begin{array}{l}L U \\
(19)\end{array}$ & $\mathbf{M}$ & -1.45 & -0.91 & $-0 \cdot 42$ & $-0 \cdot 17$ & $-0 \cdot 04$ \\
\hline $\begin{array}{l}G C \\
(4 \mathrm{q} 12 \rightarrow \mathrm{q} 13)\end{array}$ & $\begin{array}{l}\text { M } \\
\text { F } \\
\text { Sum }\end{array}$ & $\begin{array}{l}-1 \cdot 49 \\
-2 \cdot 67 \\
-4 \cdot 16\end{array}$ & $\begin{array}{l}-0.94 \\
-1.79 \\
-2.73\end{array}$ & $\begin{array}{l}-0.43 \\
-0.90 \\
-1.33\end{array}$ & $\begin{array}{l}-0 \cdot 18 \\
-0 \cdot 42 \\
-0 \cdot 60\end{array}$ & $\begin{array}{l}-0.04 \\
-0.14 \\
-0.18\end{array}$ & $\begin{array}{l}C 3 \\
(19 p 13)\end{array}$ & $\mathbf{M}$ & $-0 \cdot 19$ & $-0 \cdot 14$ & -0.08 & $-0 \cdot 03$ & $-0 \cdot 01$ \\
\hline $\begin{array}{l}M N S \\
(4 \mathrm{q} 28 \rightarrow \mathrm{q} 31)\end{array}$ & $\begin{array}{l}\mathbf{M} \\
\mathbf{F} \\
\text { Sum* }\end{array}$ & $\begin{array}{l}-0.09 \\
-0.46 \\
-2 \cdot 87\end{array}$ & $\begin{array}{l}-0 \cdot 07 \\
-0 \cdot 20 \\
-1 \cdot 43\end{array}$ & $\begin{array}{r}-0 \cdot 04 \\
0 \cdot 00 \\
-0 \cdot 67\end{array}$ & $\begin{array}{l}-0 \cdot 02 \\
+0 \cdot 05 \\
-0 \cdot 27\end{array}$ & $\begin{array}{r}0.00 \\
+0.05 \\
-0.06\end{array}$ & $\begin{array}{l}A D A \\
(20 \mathrm{q} 13 \cdot 2 \rightarrow \mathrm{qter}) \\
P I \\
(22)\end{array}$ & $\begin{array}{l}\mathbf{M} \\
\mathbf{F}\end{array}$ & $\begin{array}{l}-0.30 \\
+0.02\end{array}$ & $\begin{array}{l}-0 \cdot 19 \\
+0 \cdot 01\end{array}$ & $\begin{array}{l}-0 \cdot 08 \\
+0 \cdot 01\end{array}$ & $\begin{array}{l}-0 \cdot 03 \\
+0 \cdot 01\end{array}$ & $\begin{array}{r}-0 \cdot 01 \\
0 \cdot 00\end{array}$ \\
\hline $\begin{array}{l}M E 2 \\
(6 \mathrm{p})\end{array}$ & $\mathrm{F}$ & -1.72 & $-1 \cdot 14$ & $-0 \cdot 59$ & $-0 \cdot 30$ & $-0 \cdot 11$ & & Sum* & $-0 \cdot 07$ & -0.29 & $-0 \cdot 12$ & $-0 \cdot 03$ & $-0 \cdot 01$ \\
\hline $\begin{array}{l}H L A \\
(6 \mathrm{p} 21 \cdot 3)\end{array}$ & $\begin{array}{l}\text { M } \\
\text { F } \\
\text { Sum }\end{array}$ & $\begin{array}{l}-2 \cdot 06 \\
-1 \cdot 30 \\
-3 \cdot 36\end{array}$ & $\begin{array}{l}-1 \cdot 24 \\
-0 \cdot 76 \\
-2 \cdot 00\end{array}$ & $\begin{array}{l}-0 \cdot 52 \\
-0 \cdot 18 \\
-0 \cdot 70\end{array}$ & $\begin{array}{l}-0 \cdot 20 \\
+0 \cdot 06 \\
-0 \cdot 14\end{array}$ & $\begin{array}{r}-0.04 \\
+0.10 \\
+0.06\end{array}$ & C6 & $\begin{array}{l}\text { M } \\
\text { F } \\
\text { Sum }\end{array}$ & $\begin{array}{l}-0 \cdot 72 \\
-0 \cdot 08 \\
-0 \cdot 80\end{array}$ & $\begin{array}{l}-0.44 \\
-0.07 \\
-0.51\end{array}$ & $\begin{array}{l}-0 \cdot 19 \\
-0.05 \\
-0 \cdot 24\end{array}$ & $\begin{array}{l}-0 \cdot 08 \\
-0 \cdot 04 \\
-0 \cdot 12\end{array}$ & $\begin{array}{l}-0 \cdot 02 \\
-0 \cdot 02 \\
-0 \cdot 04\end{array}$ \\
\hline $\begin{array}{l}B F \\
(6 p 21 \cdot 3)\end{array}$ & $\mathbf{M}$ & $-2 \cdot 07$ & $-1 \cdot 25$ & $-0 \cdot 53$ & $-0 \cdot 20$ & -0.05 & $\begin{array}{l}C D A \\
F 13 B\end{array}$ & $\begin{array}{l}\mathrm{F} \\
\mathrm{M} \\
\mathrm{F}\end{array}$ & $\begin{array}{l}-2 \cdot 17 \\
-0.27 \\
+0.20\end{array}$ & $\begin{array}{l}-1 \cdot 38 \\
-0.04 \\
+0 \cdot 18\end{array}$ & $\begin{array}{l}-0.67 \\
+0.09 \\
+0.14\end{array}$ & $\begin{array}{l}-0 \cdot 35 \\
+0 \cdot 08 \\
+0 \cdot 10\end{array}$ & $\begin{array}{l}-0 \cdot 16 \\
+0 \cdot 03 \\
+0.05\end{array}$ \\
\hline $\begin{array}{l}G L O \\
(6 \mathrm{p} 21 \cdot 31 \rightarrow \mathrm{p} 21 \cdot 1)\end{array}$ & $\mathbf{M}$ & $-0 \cdot 15$ & $-0 \cdot 11$ & -0.06 & -0.03 & $-0 \cdot 01$ & & Sum & $-0 \cdot 07$ & $+0 \cdot 14$ & $+0 \cdot 23$ & $+0 \cdot 18$ & $+0 \cdot 08$ \\
\hline $\begin{array}{l}P G M 3 \\
(6 \mathrm{q} 12)\end{array}$ & $F$ & $-0 \cdot 70$ & $-0 \cdot 43$ & $-0 \cdot 19$ & $-0 \cdot 07$ & $-0 \cdot 02$ & $G P T$ & $\begin{array}{l}\text { M } \\
\text { F } \\
\text { Sum }\end{array}$ & $\begin{array}{l}-2.05 \\
-1.40 \\
-2.45\end{array}$ & $\begin{array}{l}-1 \cdot 24 \\
-0.84 \\
-2.08\end{array}$ & $\begin{array}{l}-0 \cdot 52 \\
-0 \cdot 31 \\
-0 \cdot 83\end{array}$ & $\begin{array}{l}-0.20 \\
-0.07 \\
-0.27\end{array}$ & $\begin{array}{l}-0 \cdot 04 \\
+0 \cdot 02 \\
-0 \cdot 02\end{array}$ \\
\hline
\end{tabular}

* Including the data of Eastman et al. ${ }^{5}$

stigmata. Blood samples were analysed for a total of 36 marker loci, of which 27 were informative for linkage studies (table). $K E L, C O, K m, A H C Y, A K$, $A M Y 2, G A L T, G O T 1$, and $P G D$ were not informative. Linkage studies were performed using the LIPED 3 computer programme of $\mathrm{Ott}^{\mathrm{\gamma}}$; a penetrance of $97 \%$ was used for the analysis.

\section{Results and discussion}

The part of the pedigree analysed is shown in the figure, together with the relevant clinical data. Fourteen of the 19 family members investigated had symptoms of Van der Woude syndrome, six males and eight females. Ten of them had lip pits and the
TABLE (continued)

other four had some evidence of cleft lip or palate. Another five subjects not seen by us were reported to have lip pits or other manifestations (II.1, III.1, III.4, IV.1, and V.2).

The lod scores at standard recombination fractions are given for each locus analysed in the table, along with the scores of Eastman et al. ${ }^{5}$ Uniformly negative lod scores were obtained with $A C P 1$, $A D A, B F, C 3, C 6, C D A, E S D, G C, G L O, G m$, GPT, HLA, LU, ME2, MNS, PGM1, PGM3, PI, $P L G, R h$, and $T F$. Inconclusive but largely negative lod scores were obtained with $A B O, F 13 B, J K$, $H P$, and $P 1$. Only the $V W S: F y$ relation resulted in uniformly positive lod scores: $\hat{\theta}=0 \cdot 0, \mathrm{z}(\hat{\theta})=1 \cdot 31$. After combining our data with those of Eastman $e t$ 
$a l,{ }^{5}$ however, a peak lod score at $\hat{\theta}=0 \cdot 09, \mathrm{z}(\hat{\theta})=1 \cdot 13$ for VWS:Fy was calculated. Further studies are being undertaken to verify the possible Duffy linkage.

\section{We thank the Deutsche Forschungsgemeinschaft for financial support.}

\section{References}

' Van der Woude A. Fistula labii inferioris congenita and its association with cleft lip and palate. Am J Hum Genet 1954:6:244-56.

2 Cervenka J, Gorlin R, Anderson V. The syndrome of pits of the lower lip and cleft lip and/or palate. Genetic considerations. Am J Hum Genet 1967;19:416-32.

3 Janku P, Robinow M. Kelly T. Bradley R, Baynes A, Edgerton MT. The van der Woude syndrome in a large kindred: variability, penetrance, genetic risks. $A m$ J Med Gener 1980;5:117-23.
${ }^{4}$ Schneider EL. Lip pits and congenital absence of second premolars: varied expression of the lip pits syndrome. J Med $\overrightarrow{\overline{\vec{D}}}$ Genet 1973;10:346-9.

5 Eastman JR, Bixler D. Escobar V. Linkage studies in Van der Woude syndrome. J Med Genet 1978:15:217-8.

' Spence MA, Glass L. Miles J. Field LL. Sparkes R, Stewart R. Linkage analysis with van der Woude syndrome. Cytogenet Cell Genet 1982;32:321.

${ }^{7}$ Hudek G. Untersuchungen an zwei Stammbäumen mit dominant vererbten Spaltbildungen im Lippen-Kiefer-Gaumen-Bereich. Dissertation. Medizinische Fakultät der Universität Freiburg, $\vec{\circ}$ 1985.

* Ott J. Estimation of recombination fraction in human pedigrees. Efficient computation of the likelihood for human linkage studies. Am J Hum Genet 1974;26:588-97.

Correspondence and requests for reprints to $\operatorname{Dr} \mathrm{K}$ Bender, Institut für Humangenetik und Anthropolo- $\omega$ gie der Universität Freiburg, Albertstrasse 11, $\vec{\circ}$ D-7800 Freiburg, West Germany. 